\title{
PROGRAMA PARA EL CONTROL DEL ESTRÉS APLICADO A UNA VÍCTIMA DE MOBBING: ESTUDIO DE UN CASO
}

\author{
STRESS MANAGEMENT PROGRAM APPLIED TO A MOBBED WORKER: STUDY \\ OF A CASE
}

\author{
David González-Trijueque \\ Tribunal Superior de Justicia de Madrid, España
}

\begin{abstract}
Resumen: Se describe el tratamiento y evolución de una mujer acosada en su lugar de trabajo, que presenta sintomatología ansioso-depresiva. Se utilizó un programa de control del estrés (Robles \& Peralta, 2011). La evaluación se realizó en pre-tratamiento, post-tratamiento y seguimientos de uno, tres y seis meses. El tratamiento constó de catorce sesiones en siete semanas de noventa minutos por sesión. Los objetivos del tratamiento se establecieron a partir de variables sintomatológicas, evaluadas a partir del SCL-90-R (González de Rivera, De las Cuevas, Rodríguez-Abuín \& Rodríguez-Pulido, 2002). El propósito fue capacitar a la paciente para disminuir su malestar psicológico en el trabajo y afrontar de forma más satisfactoria el estresor laboral que identificaba como una situación de acoso laboral. Después del tratamiento, en las instancias de seguimiento, continuaba con su recuperación; los resultados sugieren una mejoría significativa a corto y largo plazo en las medidas realizadas. Por último, se discuten los resultados de la intervención, que apuntan hacia la utilidad del programa aplicado, la cual deberá ser corroborada en estudios posteriores.
\end{abstract}

Palabras Clave: mobbing, acoso laboral, estrés laboral, eficacia terapéutica

\begin{abstract}
In this paper the treatment and evolution of a woman subject to mobbing in the workplace-and also exhibited anxious-depressive symptoms - is described. A stress management program was used (Robles $\&$ Peralta, 2011). The subject was assessed prior to and after treatment, with follow-up evaluations one, three and six months after treatment. The treatment consisted of fourteen 90 minutes sessions in a period of seven weeks. The treatment was designed based on the psychopathological factors assessed with the Spanish adaptation of SCL-90-R (González de Rivera, De las Cuevas, Rodríguez-Abuín \& Rodríguez-Pulido., 2002). The treatment was aimed at training the subject to reduce her psychological discomfort in the workplace and to cope more efficiently with the situation perceived as work harassment. After the treatment process and at one month, at three months and six months follow ups, the patient showed a complete recovery; in fact, the obtained results suggest a significant improvement in short and long term in all of the assessed dimensions. Finally, the results of the therapeutic intervention, and its use as an applied program are discussed. Further studies will be needed to confirm the observed results.
\end{abstract}

Keywords: mobbing, workplace bullying, job stress, therapeutic efficacy

\section{Introducción}

En la actualidad, el acoso psicológico en el lugar de trabajo (mobbing) está considerado como uno de los principales riesgos psicosociales a los que puede enfrentarse un trabajador durante su vida laboral (Moreno-Jiménez \& Rodríguez-Muñoz, 2006). De hecho, el mobbing ha sido descrito como una forma específica de estrés laboral que configura un tipo de violencia psicológica que tiene lugar en el ámbito del trabajo y que puede manifestarse a través de comportamientos muy variados que se producen de forma sistemática y prolongada en el tiempo contra una persona (Einarsen, 2000; Einarsen, Hoel, Zapf \& Cooper, 2011).

Los efectos negativos para la salud del trabajador, tanto física como psicológica, del estrés laboral ya han sido constatados a través de numerosos estudios (Mateo, 2013), sin embargo, la respuesta de estrés no es más que nuestra forma de afrontar y adaptarnos a las diversas demandas o situaciones que la vida nos va imponiendo. Por ello, nuestra respuesta al estrés no es sólo fundamental para dicha adaptación, sino que gracias a ellas hemos sobrevivido como especie (Robles \& Peralta, 2011). En este sentido, debemos señalar que para determinar si un

Correspondencia: David González-Trijueque. Tribunal Superior de Justicia de Madrid, España.

Correo Electrónico: david_gonzalez@madrid.org 
estímulo es estresante o no, debemos tomar en consideración la respuesta que el individuo da frente al mencionado estímulo. Pero la realidad es que esta respuesta no depende sólo del estímulo, sino que, en gran medida, está determinada por las características del individuo que lo hacen más o menos vulnerable a la situación (Lazarus \& Folkman, 1986; Robles \& Peralta, 2011). Podemos así afirmar que la respuesta depende del significado que la situación tiene para el individuo y de la importancia que éste le concede (Mateo, 2013). A este respecto, se debe señalar que las relaciones interpersonales en el lugar de trabajo pueden suponer un importante riesgo psicosocial y que en determinados casos pueden derivar en una situación de acoso laboral, fenómeno claramente estresante para cualquier trabajador (Einarsen et al., 2011).

En el presente artículo se presenta el caso de una paciente que muestra quejas variadas que le generan malestar psicológico, entre las que destaca la sintomatología ansioso-depresiva de carácter reactivo a un estresor del ámbito laboral, que identifica como una situación de acoso hacia su persona. En términos nosológicos, la paciente presenta un F43.22 trastorno adaptativo mixto de tipo ansioso-depresivo [309.28] (American Psychiatric Association [APA], 2005), con predominio de la clínica ansiosa. A la vista de la sintomatología descrita y considerando el acoso laboral percibido como una forma específica de estrés laboral, se optó por intervenir en el caso a través de un programa para el control del estrés (Robles \& Peralta, 2011), abordaje terapeútico elegido por los buenos resultados que ha mostrado en relación a supuestos relacionados con la respuesta al estrés y que cuenta con el aval que supone el empleo de técnicas cognitivas, fisiológicas y conductuales para abordar la sintomatología generada a partir de este tipo de supuestos (Mateo, 2013). El programa de aforntamiento de estrés propuesto está organizado en tres partes, principalmente: 1) objetivar el estresor, en este caso una supuesta situación de acoso laboral; 2) establecer los objetivos del programa, los cuales lógicamente están dirigidos a controlar la respuesta de estrés producida; y 3 ) poner en marcha un plan de actuación estructurado en distintos módulos que incluyen estrategias cognitivas, fisiológicas y conductuales (Robles \& Peralta, 2011).

\section{Marco Teórico}

El mobbing es un fenómeno de origen claramente multicausal (Einarsen \& Hauge, 2006; Zapf, Einarsen, Hoel \& Vartia, 2003) que habitualmente genera importantes daños en la salud física, psíquica y social de quien lo sufre (Einarsen \& Mikkelsen, 2003; González de Rivera \& Rodríguez-Abuín, 2006; Hogh, Mikkelsen \& Hansen, 2011; Leymann \& Gustafsson, 1996; Matthiesen \& Einarsen, 2001, 2004; Mikkelsen \& Einarsen, 2001, 2002a, 2002b).

En un principio, las víctimas de acoso laboral suelen presentar confusión acerca de lo que les está sucediendo, comenzando paulatinamente a sufrir una respuesta de estrés que suele acompañarse de miedo y aislamiento (Hirigoyen, 1999, 2001; Piñuel, 2001, 2003). Este desequilibrio psicológico del trabajador afectado irá en aumento a medida que se mantiene la situación de acoso, emepezando a aparecer reacciones emocionales como ansiedad, depresión y quejas psicosomáticas (Hogh et al., 2011; Leymann, 1990). Así por ejemplo, González de Rivera y RodríguezAbuín (2006) indican que el acoso psicológico en el contexto laboral produce una severa psicopatología de instauración progresiva y que tiende a evolucionar hasta cronificarse, siendo la ansiedad y el paranoidismo muy evidentes, así como muy habituales la sintomatología depresiva y las quejas somáticas, detectándose una evidente correlacion entre el acoso percibido y la sintomatología posteriormente desarrollada (Hogh et al., 2011).

En otro estudio destacado realizado con víctimas de mobbing en Irlanda, O'Moore, Seigne, McGuire y Smith (1998) hallaron que todas ellas presentaban problemas de irritabilidad, ansiedad, sentimientos de depresión y un estado de hipervigilancia y desconfianza, siendo también muy habituales los problemas de autoestima así como un amplio rango de somatizaciones. En la misma línea, Einarsen, Matthiesen y Skogstad (1998), a través de una muestra representativa de enfermeras que se percibían acosadas en su lugar de trabajo, encontraron elevados indicadores de burnout, insatisfacción laboral y bajos niveles de bienestar psicológico.

Asimismo, muchos trabajadores acosados en su lugar de trabajo presentan sintomatología de carácter postraumático, como es la reexperimentación de la situación de acoso, sintomatología ansioso-depresiva, somatizaciones, irritabilidad, apatía, déficit de atención, 
falta de autoestima, fobia social, problemas de sueño, pesadillas y desconfianza hacia el entorno, indicadores todos ellos que sugieren una sintomatología propia del trastorno por estrés postraumático (TEPT) (Hogh et al., 2011). Esta sintomatología compatible con la existencia de un TEPT ha sido detectada en víctimas de acoso laboral en estudios realizados en Alemania (Zapf, Knorz \& Kulla, 1996), Austria (Niedl, 1996), Finlandia (Vartia, 1996), Irlanda (O'Moore et al., 1998), Noruega (Einarsen \& Raknes, 1997) y Suecia (Leymann \& Gustafsson, 1996). No obstante, se debe señalar que para poder diagnosticar un TEPT la persona debe haber sido expuesta a un acontecimiento de tal magnitud que su integridad física o la de otros se haya visto amenazada (APA, 2005), situación que no se produce habitualmente en los supuestos de mobbing, donde es la violencia psicológica la más utilizada; por ello, hay autores que consideran que se deben considerar otras etiquetas diagnósticas que no sean el TEPT pero dejando constancia de la sintomatología postraumática que se detecte (Mikkelsen \& Einarsen, 2002a).

En todo caso, el síndrome clínico propio asociado a situaciones de acoso laboral no deja de ser una respuesta adaptativa ante un estresor externo que suele derivar en algún tipo de cuadro psicopatológico, ya que son pocos los afectados que pueden mantener un correcto equilibrio psicológico sin recibir atención especializada (González de Rivera, 2003).

En el caso presentado, la paciente muestra un trastorno adaptativo mixto de tipo ansiosodepresivo, cuadro de carácter reactivo perfectamente compatible con un supuesto de mobbing; de hecho, se ha descrito al estrés como el agente etiológico fundamental del trastorno adaptativo (APA, 2005), si bien existen diversas variables y modificadores que determinan quién padecerá un trastorno adaptativo tras un acontecimiento estresante (Strain, Newcorn, Wolf \& Fulop, 2009).

La característica esencial de este tipo de trastorno es el desarrollo de síntomas emocionales o comportamentales en respuesta a un estresante psicosocial identificable (APA, 2005); estos síntomas deben presentarse durante los tres meses siguientes al inicio del estresor, tal y como sucede en el caso presentado. Además, la expresión clínica de la reacción experimentada consiste en un acusado malestar o en un deterioro significativo de la actividad social o profesional (APA, 2005). Por definición, un trastorno adaptativo debería resolverse dentro de los seis meses que siguen a la desaparición del estresor (o de sus consecuencias), por lo que el pronóstico de la paciente sería favorable siempre y cuando desaparezca la situación de acoso y el trastorno adaptativo no se convierta en una entidad diagnóstica más grave (e.g. TAG). Sin embargo, los síntomas pueden persistir por un período prolongado de tiempo (más de seis meses) si aparecen en respuesta a un estresor crónico o a un estresor con repercusiones importantes. En el caso presentado, la paciente no dejó en ningún momento de acudir a su lugar de trabajo, por lo que el estresor siempre estuvo presente durante la intervención realizada y el posterior seguimiento. Según toda la información manejada en el presente caso, el trastorno presentado era de carácter crónico en el sentido de que los síntomas persistían por más de seis meses (APA, 2005). En este tipo de cuadro clínico, el malestar o el deterioro de la actividad asociado se manifiesta frecuentemente a través de una disminución del rendimiento en el trabajo y con cambios temporales en las relaciones sociales (APA, 2005).

El tratamiento de los trastornos adaptativos se basa, fundamentalmente, en las medidas psicoterapéuticas que permiten una reducción del estrés, en mejorar el manejo del elemento estresante si no es eliminable o reductible y en establecer un sistema de apoyo para potenciar la adaptación al máximo (Strain et al., 2009). Dentro de los abordajes psicoterapéuticos que han mostrado su eficacia a la hora de intervenir con las víctimas de mobbing destacan los de tipo cognitivo-conductual (Schwickerath \& Zapf, 2011), así como el counselling en aquellos casos donde la psicopatología no resulte incapacitante para el trabajador (Tehrani, 2011). Así por ejemplo, en Alemania, Schwickerath y Zapf (2011) proponen un tipo de intervención cognitivo-conductual con trabajadores acosados que contempla el distanciamiento del problema, la comprensión del mismo, la toma de decisiones y la disposición para actuar, destacando la utilidad del análisis funcional como técnica de evaluación.

Teniendo en cuenta que, el abordaje cognitivo-conductual se ha mostrado útil a la hora de abordar las psicopatologías reactivas asociadas al acoso laboral (Schwickerath \& Zapf, 2011; Tehrani, 2003) y considerando que los abordajes terapéuticos orientados a modificar los síntomas y las conductas patológicas -sin incidir en la personalidad ni en las motivaciones-, se han mostrado 
eficaces para intervenir en situaciones relacionadas con la respuesta de estrés (Hirigoyen, 1999); la unidad clínica de la facultad de psicología de la Universidad de Granada (España) puso en marcha un programa para controlar el estrés. Dicho programa se ha desarrollado a lo largo de ocho años, como servicio asistencial, que ha ofrecido buenos resultados al respecto (Peralta, Robles, Navarrete \& Jiménez, 2009; Robles \& Peralta, 2011). Los grupos que participaron del programa fueron formados por personas sanas trabajadores de la universidad con alto nivel de estrés, con evaluaciones pre y postratamiento. Participaron 52 personas (40 mujeres y 12 hombres), distribuidas en 8 grupos. Profesores y becarios conformaron el $63,33 \%$ de la muestra, el personal administrativo un $20 \%$ y el $16,66 \%$ eran alumnos, todos ellos con una edad media de 38,4 años $(D T=9,46)$ y 17,76 años de escolaridad $(\mathrm{DT}=3,31)$. Los resultados obtenidos mostraron un importante cambio en las distintas variables evaluadas (ansiedad, depresión, hostilidad, optimismo, síntomas somáticos, estrés percibido). Se encontraron diferencias significativas en depresión, ansiedad, estrés percibido, cantidad de situaciones estresantes percibidas, disminución de la hostilidad y aumento del optimismo (Robles \& Peralta, 2011). Las personas que realizaron el programa mejoraron física y psicológicamente, aumentando su optimismo.

Este programa parte de la base que al estresor en cuestión, en este caso el acoso laboral, le sigue la percepción del mismo y la correspondiente respuesta de estrés (alarma, resistencia, agotamiento), siendo a posteriori cuando aparecen las consecuencias en la persona afectada (Robles \& Peralta, 2011). En este sentido, es fundamental contemplar el efecto modulador de las estrategias de afrontamiento (Lazarus \& Folkman, 1986), pese a que se debe tener claro que no existen estilos de afrontamiento buenos o malos per se, sino que dependerán del propio estresor a afrontar y de la propia personalidad del sujeto (Robles \& Peralta, 2011).

Dado los buenos resultados del programa, se optó por utilizar este tipo de intervención con la paciente atendida.

A continuación, se facilita el abordaje terapéutico del caso presentado, así como los resultados obtenidos.

\section{Método}

\section{Identificación del paciente y motivo de consulta ${ }^{1}$}

La paciente es una mujer de treinta y tres años, soltera y con pareja estable desde hace más de seis años. Desde hace dos años reside junto a su compañero sentimental en Madrid, manifestando un adecuado grado de adaptación a dicho modelo de convivencia. Describe sus relaciones socio-familiares y de pareja como muy satisfactorias y al momento de la exploración goza de buen estado de salud, no habiendo tenido en su historial médico ninguna enfermedad de relevancia clínica. Refiere nivel socio-económico medio-alto y posee estudios universitarios como licenciada en ciencias empresariales. Laboralmente está en activo y trabaja como profesora asociada en la facultad de ciencias económicas de una universidad privada, trabajo en el que lleva más de dos años actividad sin incidencias significativas hasta los últimos ocho meses.

La paciente acude a consulta debido al malestar psicológico que experimenta en los últimos meses. Describe sintomatología ansiosodepresiva que asocia a una situación de acoso laboral hacia su persona por parte de un superior jerárquico dentro de su departamento universitario. Informa que desde que se presentara ocho meses antes al concurso correspondiente para optar a una plaza de profesora titular, el responsable del departamento al que pertenece comenzó a tratarla despectivamente de forma sistemática, situación que la paciente achaca a que su superior no contaba con que ella fuera a optar a dicha plaza ("contaban con asignar esa plaza a otra persona [...] pensé que podría tener opciones y por eso me presenté"). La situación de acoso que refiere sufrir desde entonces ha incidido en su salud psíquica (con predominio de sintomatología ansioso-depresiva que ha derivado en el diagnóstico de un trastorno adaptativo mixto según documentación aportada), sin embargo no es partidaria de seguir tratamiento psicofarmacológico de tipo ansiolítico ni antidepresivo, pese a que se lo ha recetado su médico de atención primaria (MAP) del Centro de Salud correspondiente.

Hasta entonces, la paciente no había intentado solucionar su problema ("creía que yo

\footnotetext{
1. Se recabó autorización por escrito a la paciente para realizar el presente estudio y publicación. No obstante, los datos no relacionados con aspectos técnicos del caso, han sido modificados para impedir la identificación de la paciente.
} 
podría sola con esto"), no identificando otras situaciones de su vida que le generen ansiedad. El nivel de motivación de la paciente era muy alto, mostraba auténticos deseos de poder superar su problema y afrontaba la intervención terapéutica entendiéndola como una ocasión para conseguir mayor auto-control y poder controlarse de forma más satisfactoria en su entorno laboral, con menor afectación en su vida.

\section{Historia del problema}

La paciente afirma que nunca había presentado problemas de ansiedad o malestar psicológico durante su evolución psicobiográfica, y que estos problemas han surgido únicamente en los últimos meses a raíz de la conducta de su superior jerárquico que valora como una situación de acoso laboral hacia su persona. Describe que su malestar durante los primeros meses se centraba en síntomas de ansiedad como nerviosismo e irritabilidad y que con el paso de las semanas comenzó a tener problemas de conciliación y mantenimiento del sueño. En las últimas seis semanas ha comenzado a sufrir con mayor frecuencia cefaleas y molestias gastrointestinales. Asimismo, reconoce que en las últimas semanas se encuentra más triste y desesperanzada con su situación laboral, a la que no ha sabido hacer frente según ella misma refiere.

La paciente desea someterse a tratamiento porque entiende que no puede renunciar a su trabajo y porque considera que ha de poder tener un mayor control sobre sus respuestas de ansiedad ante la situación de estrés laboral que está viviendo. La necesidad de sentirse eficaz a la hora de controlar sus reacciones físicas y mentales, en las circunstancias que le resultaban tan desagradables, fue un factor motivacional muy importante para la intervención terapéutica desarrollada ("lo que no quiero es que me den la baja laboral, quiero intentar luchar contra esto"). Nunca antes había solicitado tratamiento psicoterapéutico para este problema ni para ningún otro. La motivación que manifiesta hacia el tratamiento es muy alta, ya que, según refiere, desea gestionar de forma más efectiva su problemática laboral. Cuenta, además, con el apoyo y la colaboración de su pareja y su entorno familiar.

\section{Instrumentos}

El análisis de los resultados corroboró que la sintomatología de la paciente era de carácter reactivo a un estresor laboral claramente identificado como una situación de acoso hacia su persona. Se obtuvo información sobre el origen y mantenimiento de la conducta problema mediante entrevistas y distintos auto-informes. La información obtenida a partir de una entrevista autobiográfica indica que el estado de salud general de la paciente es normal, no habiendo sufrido problemas de salud significativos durante su vida. Valora satisfactoriamente sus relaciones familiares. Se observa que las respuestas de malestar psicológico están plenamente relacionadas con su entorno laboral, y más concretamente a la relación de trabajo con un superior jerárquico, descartándose otros estresores que pudieran explicar la sintomatología así como otras concausas que pudieran agravar su estado.

Asimismo, se administró de forma autoaplicada un instrumento específico de medida del acoso psicológico en el lugar de trabajo, el Leymann Inventory of Psychological Terrorization -LIPT-60- o Cuestionario de estrategias de acoso en el trabajo (modificadción de González de Rivera \& Rodríguez-Abuín, 2005). Se trata de un cuestionario escalar auto-administrado que objetiva y valora sesenta diferentes estrategias de acoso que el sujeto debe valorar en una escala tipo Likert desde 0 (no presente en absoluto) hasta 4 (mucho o extremadamente presente), permitiendo obtener tres parámetros de evaluación global: 1) número total de estrategias de acoso psicológico percibido (NEAP), 2) índice global de acoso psicológico (IGAP), y 3) índice medio de acoso psicológico (IMAP); asimismo, el LIPT-60 permite obtener seis escalas secundarias que describen diferentes estrategias de acoso laboral: 1) desprestigio laboral (DL), 2) entorpecimiento del progreso (EP), 3) bloqueo de la comunicación (BC), 4) intimidación encubierta (IE), 5) intimidación manifiesta (IM), y 6 ) desprestigio personal (DP).

En cuanto al problema motivo de consulta se administró la adaptación española de una escala de screening psicopatológico, el SCL90-R de Derogatis (González de Rivera, De las Cuevas, Rodríguez-Abuín \& Rodríguez-Pulido, 2002). Se trata de un inventario de noventa síntomas cuya presencia el sujeto debe valorar en una escala tipo Likert desde 0 (no presente en absoluto) hasta 4 (mucho o extremadamente presente). Los indicadores de psicopatología del instrumento son tres índices generales: 1) índice global de gravedad de los síntomas (GSI), que evalúa la intensidad total de psicopatología; 2) 
total de síntomas positivos (PST), número de síntomas con puntuación distinta de cero; y 3) índice de síntomas positivos (PSDI), que evalúa de forma combinada la intensidad y la frecuencia de los síntomas. Además, el SCL-90-R permite obtener información sobre nueve dimensiones clínicas: 1) somatización (SOM), 2) obsesióncompulsión (OC), 3) sensibilidad interpersonal (SI), 4) depresión (DEP), 5) ansiedad (ANS), 6) hostilidad (HOS), 7) ansiedad fóbica (FOB), 8) ideación paranoide (PAR) y 9) psicoticismo (PSI) (González de Rivera et al., 2002).

\section{Impresión diagnóstica}

Tras integrar la información recabada durante la fase de evaluación, se confirma que la paciente cumple los criterios para el diagnóstico de un F43.22 trastorno adaptativo mixto, con ansiedad y estado de ánimo depresivo [309.28] (Eje I del DSM-IV-TR) (APA, 2005), ya que se dan las siguientes condiciones: 1) la aparición de síntomas emocionales o comportamentales en respuesta a un estresor identificable dentro de los tres meses siguientes a la aparición del mismo, 2) los síntomas presentes suponen un deterioro significativo en la actividad social y laboral, 3) la alteración relacionada con el estrés no cumple los criterios diagnósticos para otro trastorno específico del Eje I y no constituye una simple exacerbación de un trastorno pre-existente en el Eje I o el Eje II, y 4) los síntomas no responden a una reacción de duelo. En este caso, el trastorno adaptativo presente en la paciente es de tipo mixto, con manifestaciones predominantes de sintomatología ansioso-depresiva. Además, al presentar malestar durante más de seis meses podemos hablar de un trastorno crónico.

\section{Análisis y descripción de la conducta problema}

Terminada la fase de evaluación se llevó a cabo una explicación detallada y clara sobre el problema descrito y sobre los diversos factores que lo mantienen. En términos cognitivosconductuales, los resultados de la evaluación y el análisis de los cuestionarios fue coherente con la demanda de la paciente y la conducta problema estaba circunscrita al entorno laboral, si bien cada vez el malestar estaba incidiendo más en otras esferas de la paciente.

La paciente sitúa el inicio de su problemática hace ocho meses, momento en el que concursó a una plaza de profesora titular en la universidad donde trabaja. En ese momento su superior se sintió ignorado por ella ("no le dije que me iba a presentar a esa plaza, él contaba con dársela a otra persona menos preparada que yo y me dijo que no se esperaba eso de mí"), manifestándole a la paciente que se arrepentiría de su modo de actuar ("me sentí amenazada"). Desde entonces la paciente percibe entorpecimiento en su progreso laboral y aislamiento dentro de su entorno de trabajo que está incidiendo en su bienestar psicológico. Inicialmente consideró que no necesitaría ayuda profesional alguna pero comenzó a percibir mayor nerviosismo y problemas para conciliar el sueño. Con el paso de las semanas el trato de su superior se ha vuelto más duro hacia ella ("me ha llegado a gritar y me trata de un modo despectivo"), reconociendo un empeoramiento en su estado emocional así como aparición de sentimientos de tristeza. Al momento de acudir a consulta, la paciente está contemplando la posibilidad de solicitar la baja laboral a su médico de atención primaria ("pero quiero evitar que me den la baja [...] tampoco quiero que me mande pastillas, sé que tengo que afrontar mejor mi situación laboral para que no me haga tanto daño"). Desde los primeros episodios descritos hasta el momento antes del tratamiento, el malestar psicológico y la situación laboral no ha mejorado, configurando un problema crónico.

Los estímulos antecedentes (E) de la respuesta de ansiedad y malestar psicológico son fundamentalmente de tipo ambiental (entorno laboral) y situacional (tener que hablar con su superior). Por otra parte, las conductas problemas $(R)$ son principalmente de tipo fisiológico (taquicardia, aumento de la presión arterial, sudoración, cefaleas), cognitiva (preocupación y pensamientos negativos) y motoras (conductas evitativas). La principal consecuencia de la respuesta (C) que contribuye a su mantenimiento es el cese del malestar que consigue a través de conductas evitativas, este comportamiento refuerza negativamente la conducta problema (R-), produciéndose un aprendizaje por condicionamiento operante (Pérez-Álvarez, 1999). Existe, además, refuerzo positivo en forma de atención social por parte de su entorno $(\mathrm{R}+)$. Se observa que el problema se ha cronificado con el tiempo, permaneciendo invariable la frecuencia e intensidad de la ansiedad, sus componentes, así como los estímulos generadores de la respuesta ansiógena. A todo ello habría que añadir 
la anticipación de las posibles consecuencias negativas futuras (despido laboral, temor a un empeoramiento) y el recuerdo de las situaciones anteriores en que se ha enfrentado a su superior que hacen que la paciente hasta el momento del tratamiento se perciba con ausencia de control sobre su problema (García-Vera, 1999).

Con todo ello, la conducta problema produce un malestar significativo, al que se une la sensación de pérdida de control e ineficacia, por no considerarse capaz de afrontar situaciones que considera que la mayoría de sujetos realiza con normalidad.

\section{Objetivos}

El objetivo inicial del programa de control del estrés propuesto (Robles \& Peralta, 2011) para abordar el caso es lograr que la paciente sea capaz de controlar la respuesta de estrés y de este modo potenciar su calidad de vida y la satisfacción con ella. De este modo, se propone como objetivo terapéutico entrenar a la paciente en competencias de afrontamiento al estrés y que ésta logre un mayor control sobre las respuestas ante acontecimientos desagradables.

\section{Procedimiento}

En las primeras entrevistas se recabó la información necesaria para contextualizar el caso. Asimismo, se administraron los reactivos seleccionados para ver si la paciente realmente percibía una situación de acoso laboral (LIPT$60)$, así como para registrar su malestar psicológico (SCL-90-R). Posteriormente, se explicó a la paciente que se iba a implementar para su tratamiento un programa del control del estrés (Robles \& Peralta, 2011) estructurado en siete módulos, con dos sesiones intersemanales. Se le explicó que el número de sesiones que componen cada módulo es variable (de 1 a 4), dependiendo de los contenidos a desarrollar. Asimismo, se enfatizó en el hecho de que la mayoría de los pacientes logran una mejoría ante este tipo de problemas siguiendo las recomendaciones terapéuticas oportunas.

A continuación se expone de forma resumida el procedimiento llevado a cabo con la paciente:

- Módulo teórico de conceptualización del estrés (1 sesión): En la primera sesión se presenta el programa y se atienden las expectativas de la paciente. Se insiste en que el objetivo del programa es desarrollar un programa de entrenamiento en competencias de afrontamiento del estrés (para conseguir un mayor control sobre las respuestas de estrés), así como enseñar estrategias generales para aplicar no solamente a la problemática laboral actual sino a aquellas dificultades que puedan surgir en el futuro y potenciar la calidad de vida y la satisfacción con ella. En la segunda parte de la sesión se desarrollan explicaciones sobre conceptos tales como la diferencia entre la ansiedad normal y la patológica, el estrés laboral y más específicamente el acoso psicológico en el lugar de trabajo.

- Módulo de técnicas de desactivación (2 sesiones): En la primera sesión de este módulo se abordan aspectos, tanto teóricos como prácticos, relacionados con la respiración diafragmática (Delgado, 2014) y el entrenamiento autógeno (Schultz, 1969). La paciente se ejercita en ambas técnicas y se analiza la experiencia. Se establece practicar en casa lo aprendido, con anotaciones en auto-registro. En la segunda sesión del módulo se inicia a la paciente en técnicas de relajación muscular profunda y técnicas de imaginación temática (Davis, MCKay \& Eshelman, 2009), explicando ambas técnicas y poniéndolas en práctica, analizando la experiencia e indicando ejercitar lo aprendido fuera del entorno terapéutico, con registro de dicha actividad.

- Módulo de técnicas cognitivas (4 sesiones): En la primera sesión se analizan aspectos relacionados con la reestructuración cognitiva de la paciente, especialmente las distorsiones o errores del pensamiento (Clark \& Beck, 2012). En la segunda sesión se continúa trabajando puntos de reestructuración cognitiva de la paciente, en esta ocasión las ideas irracionales; mientras que en la tercera se debaten los pensamientos relacionados con la problemática laboral que presenta la paciente. En la cuarta sesión fijada de este módulo se facilitan auto-instrucciones de utilidad y estrategias de parada del pensamiento (Davis et al., 2009). En todas las sesiones se facilitan consideraciones teóricas y se realizan ejercicios prácticos, así como se asignan tareas para casa que deben registrase en modelos de auto-registro elaborados ad hoc.

- Módulo de técnicas de asertividad (2 sesiones): En la primera sesión se explica el comportamiento asertivo con énfasis en la importancia de las relaciones interpersonales como fuente de estrés (Mateo, 2013) así como en las habilidades sociales (Caballo, 2010). Se explica comportamiento agresivo, pasivo y asertivo, se 
asignan tareas de auto-observación y registro de los tres tipos de comportamiento. Se solicita que para la siguiente sesión la paciente trajera por escrito una situación en la que trate de defender sus derechos. En la segunda sesión, se abordan técnicas sobre cómo decir no y cómo pedir un cambio de conducta. Se explica cómo pedir un cambio de conducta a otra persona y se asigna como tarea realizar una petición de cambio de conducta y ejercitar el derecho a decir no, anotándolo en auto-registro.

- Módulo sobre administración y control del tiempo (1 sesión): Se resalta la importancia de administrar bien el tiempo para reducir el nivel de estrés y se explican técnicas para conseguir una buena organización del tiempo (Davis et al., 2009), realizándose distintos ejercicios. Se asignan tareas, con auto-registro detallado de todas las actividades que hace en el día durante tres días y cuánto tiempo le dedica a cada una de ellas; asimismo, se solicita un auto-registro de planificación y clasificación de las tareas en función de prioridad.

- Módulo sobre características de personalidad y su relación con el estrés y la salud (2 sesiones): Se explica la importancia de ciertas formas de actuar (características de personalidad) y su relación con la salud y la enfermedad, y cómo estas características de personalidad se relacionan con el estrés. En la segunda sesión se analiza la importancia de la ira/hostilidad así como estrategias de control de la ira (Clark \& Beck, 2012), explicándose técnicas de reestructuración cognitiva para controlar ira/hostilidad. Se explica cómo afrontar cuando otra persona llega con nivel de ira elevado, se le anima a exponer su experiencia y sus dudas sobre la técnica y se le pide que ponga en práctica alguna técnica para controlar la ira/hostilidad propia o de los demás.

- Módulo sobre el humor y el optimismo (1 sesión): Se explica la importancia del optimismo y su relación con la salud y la calidad de vida (Delgado, 2014); además, se aborda el humor como forma de afrontamiento explicándose técnicas sobre ambos aspectos. Se anima a la paciente a plantear situaciones problemáticas y aplicar el humor, encomendándose tareas para casa que han de ser registradas.

- Módulo integrativo de todo lo visto (1 sesión): Se analizan la evolución de la paciente a los largo de la intervención y se realizan recomendaciones, fijándose sesiones posttratamiento para seguimiento.

\section{Resultados: efectividad del tratamiento y seguimiento}

Inicialmente se administró a la paciente el LIPT-60 para verificar si nos encontramos ante un supuesto compatible con una situación de acoso laboral o por el contrario se tratase de otro tipo de problemática en el lugar de trabajo. A este respecto, se debe señalar que siguiendo las consideraciones interpretativas de los autores de la prueba, nos encontraríamos ante un caso muy probablemente de acoso laboral si el centil correspondiente a la puntuación del IGAP fuese al menos 60 o en su defecto dos de las subescalas de la prueba, todo ello en relación al baremo de población acosada. En el caso de la paciente que nos ocupa nos encontramos en la segunda situación, el centil de su IGAP no alcanza 60 (IGAP=50), sin embargo sí lo hacen tres de las seis subescalas (entorpecimiento del progreso, bloqueo de la comunicación e intimidación encubierta) (Tabla 1), por lo que podemos señalar que la situación descrita es propia de un caso de mobbing.

Tabla 1. Puntuaciones directas y centiles obtenidos en el LIPT-60

\begin{tabular}{ccc}
\hline $\begin{array}{c}\text { Parámetros } \\
\text { Globales }\end{array}$ & P.D. & Centil \\
\hline NEAP & 40 & 70 \\
IGAP & 1,41 & 50 \\
IMAP & 2,13 & 35 \\
\hline Dimensiones & P.D. & Centil \\
secundarias & & \\
\hline DL & 1,50 & 30 \\
EP & 3,15 & 85 \\
BC & 2,55 & 75 \\
IE & 0,57 & $\mathbf{6 0}$ \\
IM & 1,83 & 40 \\
DP & 0,14 & 25 \\
\hline
\end{tabular}

En este caso, se trata de una situación de acoso en la que la paciente percibe un bloqueo sistemático y degradación laboral, con medidas de agravio comparativo y asignación de tareas impropias en la forma o en el contenido (EP), incomunicación tanto hacia dentro como hacia fuera de la organización (BC) y amenazas y daños encubiertos, en ocasiones sin que se pueda determinar sus responsables (IE), tratándose de una intimidación "limpia", que no deja "huella".

Tras comprobar que el supuesto evaluado se corresponde a una situación de acoso laboral y que la sintomatología referida se relacionaba con la temática del estrés laboral, se planteó a la paciente la posibilidad de realizar interven- 
ción con un programa del control del estrés, a lo que mostró su conformidad. La paciente mostró una adecuada adherencia terapéutica y se implicó en el tratamiento solicitado desde el comienzo del mismo. Su motivación y su nivel sociocultural fueron herramientas fundamentales para la consecución de los objetivos propuestos. Además, contaba con el apoyo social de sus seres más cercanos. El proceso de evaluación se llevó a cabo en cinco momentos distintos: pre-tratamiento (PRE), pos-tratamiento (POST), seguimientos tras uno (SEG-1), tres (SEG-2) y seis (SEG-3) meses.

En cuanto a los parámetros sintomatológicos generales podemos señalar que las pun- tuaciones obtenidas disminuyeron significativamente tanto a nivel de número total de síntomas (PST) como malestar general (GSI), si bien las medidas correspondientes a malestar medio sintomatológico (PSDI) no disminuyeron en la misma proporción (situación lógica atendiendo a la disminución del PST) (Tabla 2; Figura 1).

Respecto a los indicadores clínicos valorados a través de las distintas escalas secundarias del SCL-90-R se puede señalar que los niveles de ansiedad, somatización, obsesivocompulsivo, fobias y psicoticismo disminuyeron significativamente en el momento de concluir la intervención, manteniéndose los progresos obtenidos en los distintos seguimientos realizados.

Tabla 2. Resultados en el SCL-90-R en los distintos momentos de medida

\begin{tabular}{lccccc}
\hline $\begin{array}{l}\text { Dimensiones } \\
\text { generales }\end{array}$ & $\begin{array}{c}\text { PD } \\
\text { (Centil) } \\
\text { PRE }\end{array}$ & $\begin{array}{c}\text { PD } \\
\text { (Centil) } \\
\text { POST }\end{array}$ & $\begin{array}{c}\text { PD } \\
\text { (Centil) } \\
\text { SEG-1 }\end{array}$ & $\begin{array}{c}\text { PD } \\
\text { (Centil) } \\
\text { SEG-2 }\end{array}$ & $\begin{array}{c}\text { PD } \\
\text { (Centil) } \\
\text { SEG-3 }\end{array}$ \\
\hline PST & $51(95)$ & $33(65)$ & $32(60)$ & $26(45)$ & $24(40)$ \\
GSI & $1,12(90)$ & $0,54(55)$ & $0,54(55)$ & $0,45(45)$ & $0,40(35)$ \\
PSDI & $1,97(65)$ & $1,47(30)$ & $1,51(35)$ & $1,55(35)$ & $1,50(30)$ \\
& & & & & \\
\hline Dimensiones & PD & PD & PD & PD & PD \\
secundarias & (Centil) & (Centil) & (Centil) & (Centil) & (Centil) \\
& PRE & POST & SEG-1 & SEG-2 \\
\hline SOM & $1,25(80)$ & $0,50(45)$ & $0,50(45)$ & $0,33(35)$ & $0,33(35)$ \\
OBS & $0,50(50)$ & $0,20(35)$ & $0,20(35)$ & $0,20(35)$ & $0,20(35)$ \\
INT & $1,00(85)$ & $0,67(70)$ & $0,55(65)$ & $0,55(65)$ & $0,44(55)$ \\
DEP & $1,61(90)$ & $0,92(60)$ & $0,92(60)$ & $0,69(45)$ & $0,53(35)$ \\
ANS & $1,70(95)$ & $0,50(55)$ & $0,45(50)$ & $0,40(45)$ & $0,30(30)$ \\
HOS & $1,50(90)$ & $0,50(60)$ & $0,50(60)$ & $0,50(60)$ & $0,33(45)$ \\
FOB & $0,85(90)$ & $0,14(45)$ & $0,14(45)$ & $0,14(45)$ & $0,14(45)$ \\
PAR & $1,16(90)$ & $0,66(70)$ & $0,66(70)$ & $0,50(60)$ & $0,50(60)$ \\
PSI & $0,20(60)$ & $0(5)$ & $0(5)$ & $0(5)$ & $0(5)$
\end{tabular}

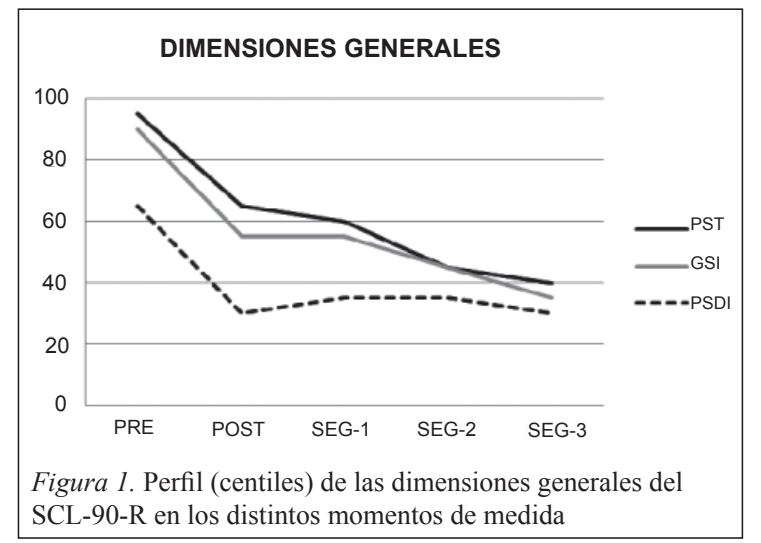

Figura 1. Perfil (centiles) de las dimensiones generales de SCL-90-R en los distintos momentos de medida
Sin embargo, la sintomatología relacionada con la depresión, las relaciones interpersonales, la hostilidad o el paranoidismo disminuyeron de forma más gradual y progresiva (Figura 2).

Un mes más tarde de finalizar las sesiones fijadas se mantuvo otra entrevista con la paciente. Ésta informó sobre una estabilidad en la mejoría alcanzada, describiendo fundamentalmente una mayor capacidad de control que le permitía no experimentar tanto malestar como el que mostraba con anterioridad a comenzar la intervención ("sigo pensando que la situación en el trabajo no es buena para mí, pero me afecta menos"). De este modo, el malestar subjetivo de la paciente había disminuido considerablemente y no se evidenciaba como incapacitante atendiendo a sus propias referencias. 
Posteriormente, se realizó un seguimiento a los tres y a los seis meses mediante una entrevista telefónica con la paciente, momento en el que se le proporcionaron algunas pautas de mantenimiento. Asimismo, se le enviaron por correo las escalas de medida anteriormente empleadas y cuyos resultados arrojaban un mantenimiento en los buenos resultados obtenidos (Tabla 2; Figuras 1 y 2). Según las propias referencias de la paciente, las quejas que motivaron recibir psicoterapia especializada habían desaparecido en gran medida y, en todo caso, percibía una mayor sensación subjetiva de control al respecto que le hacían percibir su situación como no incapacitante.

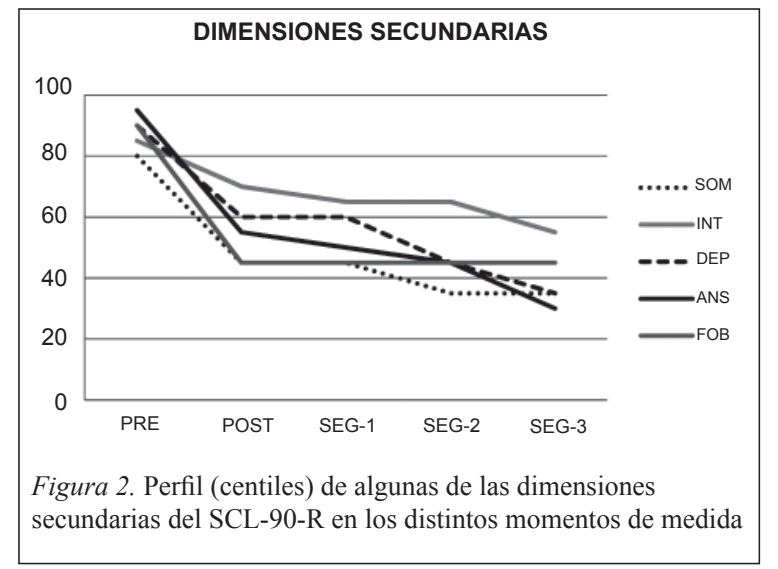

\section{Discusión}

En los últimos años, las víctimas de acoso laboral se han convertido en un supuesto habitual en la práctica psicoterapéutica (Schwickerath \& Zapf, 2011), habiéndose propuesto distintos modos de intervenir clínicamente con las víctimas de mobbing (González de Rivera, 2003; Hirigoyen, 1999, 2001; Tehrani, 2003). A la hora de intervenir con las víctimas de mobbing, resulta esencial que éstas identifiquen la situación de acoso y comprendan lo que le está sucediendo (González de Rivera, 2003; Hirigoyen, 1999), que se liberen de la culpabilidad (Hirigoyen, 1999; Piñuel, 2003), que aprendan modos de actuar ante el estresor (González de Rivera, 2003) y que ganen seguridad en sí mismas (Tehrani, 2011). Siendo el objetivo general, lograr la mejor adaptación posible y minimizar los daños sufridos (González de Rivera, 2003; Tehrani, 2011). En todo caso, lo que debe conocer la víctima de mobbing es que, siempre es posible encontrar una solución y que no todas las situaciones de acoso laboral terminan generando secuelas irreversibles en el plano psíquico del trabajador (Piñuel, 2003). Si bien existe un importante riesgo de dañar la salud mental del mismo, al menos de forma temporal, y desde luego siempre afecta al bienestar y a la calidad de vida del trabajador que lo padece (Hirigoyen, 2001; Tehrani, 2003).

Entre los estresores más habituales se encuentran las situaciones de tensión crónica mantenida en el tiempo, tal y como sucede en el caso abordado. Es sobradamente conocido que dichas situaciones de estrés prolongado pueden derivar en trastornos coronarios, respiratorios, inmunológicos, endocrinos, metabólicos, sexuales, gastrointestinales, dermatológicos, musculares, y fundamentalmente psicopatológicos (Piñuel, 2001), tal y como sucede con la paciente, quien ha llegado a presentar un trastorno adaptativo tal y como se ha señalado.

Los resultados alcanzados en el presente estudio sugieren la utilidad del programa para el control del estrés utilizado para abordar algunos supuestos de mobbing. Si bien se ha de tener en cuenta que dicha metodología ha sido aplicada con fines exploratorios y precisaría de un mayor desarrollo e investigación al respecto. Además, se deben tener en cuenta las limitaciones del estudio realizado, en el sentido de tratarse de un caso único, con una paciente con una sintomatología no incapacitante (durante todo el proceso terapéutico, ni en los meses previos precisó una baja laboral) y que gozaba de alta motivación, así como numerosas variables amortiguadoras (e. g. apoyo sociofamiliar, ausencia de patologías previas), para paliar los efectos del estresor identificado como una situación de acoso laboral. En ese sentido, se debe decir que, posiblemente, ante cuadros clínicos más graves e incapacitantes o cuadros similares desarrollados en víctimas vulnerables, los resultados obtenidos no hubieran sido tan positivos tras administrar un programa genérico de control del estrés y tal vez se precisara un abordaje más específico, centrado en el acoso psicológico en el lugar de trabajo.

En todo caso, la paciente presentó una muy positiva evolución desde el inicio de la intervención hasta la finalización de la misma. Los resultados obtenidos sugieren una mejoría en términos generales así como respecto a indicadores clínicos específicos en todas las medidas realizadas. Dicha mejoría, además, se ha mantenido relativamente estable en los periodos de seguimiento marcados $(1,3$ y 6 meses). No obstante, se debe reconocer que 
algunas medidas específicas disminuyeron de forma más significativa (e. g. ansiedad, somatización, obsesivo-compulsivo, fobias, psicoticismo), manteniéndose los progresos obtenidos en los distintos seguimientos realizados. Mientras que otros indicadores (e. g. depresión, relaciones interpersonales, hostilidad, paranoidismo) disminuyeron de forma más gradual y progresiva, situación que puede explicarse por el propio proceso de readaptación al entorno laboral una vez aplicadas las destrezas adquiridas a través del programa para el control del estrés aplicado.

No debemos olvidar que los trastornos adaptativos, en principio configuran cuadros clínicos de buen pronóstico en adultos (Strain et al., 2009) y que la intervención cognitivoconductual goza de buenos resultados ante este tipo de cuadros reactivos de origen laboral. El tratamiento instaurado, ha obtenido resultados positivos, entendiendo por éxito terapéutico la desaparición de los criterios diagnósticos del DSM-IV-TR en relación al trastorno adaptativo del que fuera diagnosticada, y el hecho de que la paciente fuera capaz de afrontar la situación temida con una baja ansiedad y sin interferencias graves en su vida diaria. Los resultados pueden considerarse satisfactorios, tomando como criterio la disminución de la sintomatología, la ausencia de sentimientos de incapacidad a la hora de desenvolverse en distintas áreas de su vida y las puntuaciones en las diferentes variables estudiadas a través del auto-informe administrado.

Los resultados alcanzados vienen a confirmar la idea de que ante los cuadros clínicos de carácter reactivo propios de las personas acosadas en su lugar de trabajo (al menos durante las primeras fases del acoso), pueden ser intervenidos desde el paradigma cognitivo-conductual en todo lo relacionado con dotar al paciente de diversas estrategias de afrontamiento y proporcionarle un mayor control sobre la situación percibida. Lógicamente, cuanto más prolongado y severo resulte el acoso, más probable es que la persona afectada termine desarrollando psicopatología más grave y este tipo de programas no se muestre tan eficaz.

En todo caso, la propuesta terapéutica planteada constituye una alternativa para abordar este tipo de supuestos (o al menos una parte de ellos), debiendo recordar que la mayoría de las personas que desarrollan algún trastorno relacionado con el estrés se recuperan por completo y pueden reincorporarse al trabajo
(Mateo, 2013). Sin embargo, esta reincorporación no está exenta de dificultades, por lo que se antoja imprescindible realizar un seguimiento para vigilar su readaptación con las habilidades y herramientas adquiridas, aunque lógicamente, si no se han corregido las causas que propiciaron la aparición de la psicopatología asociada a la situación estresante, en este caso el mobbing, la posibilidad de remisión total sintomatológica resulta poco probable.

\section{Referencias}

American Psychiatric Association (APA) (2005). Manual diagnostico y estadístico de los trastornos mentales. Texto revisado (DSM-IV-TR). Barcelona: Masson.

Caballo, V. (2010). Manual de evaluación y entrenamiento de las habilidades sociales. Madrid: Siglo XXI.

Clarck, D. A. \& Beck, A. T. (2012). Terapia cognitiva para los trastornos de ansiedad. Bilbao: Descleé de Brouwer.

Davis, M., McKay, M., \& Eshelman, E. R. (2009). Técnicas de autocontrol emocional. Barcelona: Martínez-Roca.

Delgado, S. (2014). Psicoterapia natural: recursos terapéuticos al alcance de todos. Almería: Círculo Rojo.

Einarsen, S. (2000). Harassment and bullying at work: A review of the Scandinavian approach. Aggression and Violent Behaviour, 5(4), 379-401.

Einarsen, S. \& Hauge, L. J. (2006). Antecedentes y consecuencias del acoso psicológico en el trabajo: una revisión de la literatura. Revista de Psicología del Trabajo y de las Organizaciones, 22(3), 251-274.

Einarsen, S., Hoel, H., Zapf, D. \& C. L. Cooper (2011). The concept of bullying at work: the European tradition. En S. Einarsen, H. Hoel, D. Zapf y C. L. Cooper (Coords.) Bullying and harassment in the workplace (pp. 3-40). Boca Raton, FL: CRC Press.

Einarsen, S., Matthiesen, S. B. \& Skogstad, A. (1998). Bullying, burnout and well-being among assistant nurses. Journal of Occupational Health and Safety, 14, 263-268.

Einarsen, S. \& Mikkelsen, E. G. (2003). Individual effects of exposure to bullying at work. En S. Einarsen, H. Hoel, D. Zapf y C. L. Cooper (Eds.), Bullying and emotional abuse in the workplace: international perspectives in research and practice (pp. 127-144). London: Taylor \& Francis.

Einarsen, S. \& Raknes, B. I. (1997). Harassment at work and victimization of men. Violence and Victims, 12, 247-263.

García-Vera, M. P. (1999). Análisis funcional de un caso de estrés. En F. J. Labrador, J. A. Cruzado y M. Muñoz (Coords.), Manual de técnicas de modificación y terapia de conducta (pp. 353-365). Madrid: Pirámide.

González de Rivera, J. L. (2003). El maltrato psicológico: cómo defenderse del mobbing y otras formas de acoso. Madrid: Espasa.

González de Rivera, J. L., De las Cuevas, C., RodríguezAbuín, M. J. \& Rodríguez-Pulido, F. (2002). El cuestionario de 90 síntomas (adaptación española del $S C L-90-R$ de L. R. Derogatis). Madrid: TEA.

González de Rivera, J. L. \& Rodríguez-Abuín, M. J. (2006). Acoso psicológico en el trabajo y psicopatología: un estudio con el LIPT-60 y el SCL-90-R. Revista de Psicología del Trabajo y de las Organizaciones, 22(3), 397-412.

González de Rivera, J. L. \& Rodríguez-Abuín, M. J. (2005). EI LIPT-60. Cuestionario de estrategias de acoso psicológico. Manual. Madrid: EOS. 
Hirigoyen, M. F. (1999). El acoso moral: el maltrato psicológico en la vida cotidiana. Barcelona: Paidós.

Hirigoyen, M. F. (2001). El acoso moral en el trabajo: distinguir lo verdadero de lo falso. Barcelona: Paidós.

Hogh, A., Mikkelsen, E. G. \& Hansen, A. M. (2011). Individual consequences of workplace bullying/mobbing. En S. Einarsen, H. Hoel, D. Zapf y C. L. Cooper (Coords.), Bullying and harassment in the workplace (pp. 107128). Boca Raton, FL: CRC Press.

Lazarus, R. S., \& Folkman, S. (1986). Estrés y procesos cognitivos. Barcelona: Martínez-Roca.

Leymann, H. (1990). Mobbing and psychological terror at workplaces. Violence and Victims, 5(2), 119-126.

Leymann, H. \& Gustafsson, A. (1996). Mobbing at work and the development of post-traumatic stress disorder. European Journal of Work and Organizational Psychology, 5(2), 251-275.

Mateo, P. (2013). Control del estrés laboral. Madrid: FC Editorial.

Matthiesen, S. B. \& Einarsen, S. (2001). MMPI-2 configurations among victims of bullying at work. European Journal of Work and Organizational Psychology, 10(4), 467-484.

Matthiesen, S. B. \& Einarsen, S. (2004). Psychiatric distress and symptoms of PTSD among victims of bullying at work. British Journal of Guidance and Counselling, 32(3), 335-356.

Mikkelsen, E. G. \& Einarsen, S. (2001). Bullying in Danish work-life: Prevalence and health correlates. European Journal of Work and Organizational Psychology, 10(4), 393-413.

Mikkelsen, E. G. \& Einarsen, S. (2002a). Basic assumptions and symptoms of post-traumatic stress among victims of bullying at work. European Journal of Work and Organizational Psychology, 11(1), 87-111.

Mikkelsen, E. G. \& Einarsen, S. (2002b). Relationships between exposure to bullying at work and psychological and psychosomatic health complains: The role of state negative affectivity and generalized self-efficacy. Scandinavian Journal of Psychology, 43, 397-405.

Moreno Jiménez, B. \& Rodríguez Muñoz, A. (2006). Introducción del número monográfico sobre acoso psicológico en el trabajo: una perspectiva general. Revista de Psicología del Trabajo y de las Organizaciones, 22(3), 245-249.

Niedl, K. (1996). Mobbing and wellbeing: economic and personal development implications. European Journal of Work and Organizational Psychology, 5(2), 239-249.
O’Moore, M., Seigne, M., McGuire, L. \& Smith, M. (1998). Victims of bullying at work in Ireland. Journal of Occupational Health and Safety, 14(6), 569-574.

Peralta, M. I., Robles, H., Navarrete, N. \& Jiménez, J. (2009). Aplicación de la terapia de afrontamiento al estrés en dos poblaciones con alto estrés: pacientes crónicos y personas sanas. Salud Mental, 32, 251-258.

Pérez Álvarez, M. (1999). Técnicas operantes para el desarrollo de conductas. En F. J. Labrador, J. A. Cruzado y M. Muñoz (Coords.), Manual de técnicas de modificación y terapia de conducta (pp. 477-500). Madrid: Pirámide.

Piñuel, I. (2001). Mobbing. Cómo sobrevivir al acoso psicológico en el trabajo. Santander: Sal Térrae.

Piñuel, I. (2003). Mobbing:manualde autoayuda. Madrid:Aguilar.

Robles, H. \& Peralta, M. I. (2011). Programa para el control del estrés. Madrid: Pirámide.

Schultz, J. H. (1969). El entrenamiento autógeno. Barcelona: Editorial Científico-Médica.

Schwickerath, J. \& Zapf, D. (2011). Inpatient treatment of bullying victims. En S. Einarsen, H. Hoel, D. Zapf y C. L. Cooper (Coords.), Bullying and harassment in the workplace (pp. 397-422). Boca Raton, FL: CRC Press.

Strain, J. J., Newcorn, J., Wolf, D. \& Fulop, G. (2009). Trastorno adaptativo. En R. E. Hales y S. C. Yudofsky (Coords.), Tratado de psiquiatría clínica (pp 711-721). Barcelona: Masson.

Tehrani, N. (2003). Counselling and rehabilating employees involved with bullying. En S. Einarsen, H. Hoel, D. Zapf y C. L. Cooper (Coords.), Bullying and emotional abuse in the workplace (pp. 270-284). London: Taylor \& Francis.

Tehrani, N. (2011). Workplace bullying: The role for counselling. En S. Einarsen, H. Hoel, D. Zapf y C. L. Cooper (Coords.), Bullying and harassment in the workplace (pp. 381-396). Boca Raton, FL: CRC Press.

Vartia, M. (1996). The sources of bullying-psychological work environment and organizational climate. European Journal of Work and Organizational Psychology, 5, 203-214.

Zapf, D., Einarsen, S., Hoel, H. \& Vartia, M. (2003). Empirical findings on bullying in the workplace. En S. Einarsen, H. Hoel, D. Zapf y C. L. Cooper (Eds.), Bullying and emotional abuse in the workplace: international perspectives in research and practice (pp. 103-126). London: Taylor \& Francis.

Zapf, D., Knorz, C. \& Kulla, M. (1996). On the relationship between mobbing factors and job content, social work environment and health outcomes. European Journal of Work and Organizational Psychology, 5(2), 215-237.

Para citar este artículo:

González-Trijueque, D. (2014). Programa para el control del estrés aplicado a una víctima de mobbing: estudio de un caso. Ciencias Psicológicas VIII (2): 151- 162 